\title{
Practical Tips in Neuromodulation for Pain
}

\section{Bendersky D* and Asem M \\ Department of Spine surgery and Pain Management, Clinica Monte Grande, Argentina}

*Corresponding author: Damian Bendersky, Department of Spine and Pain Management, Clinica Monte Grande, Monte Grande, Provincia de Buenos Aires, Argentina, Tel/Fax: (005411) - 4367-6700; Email: damianbendersky@hotmail.com

\section{Editorial}

Volume 3 Issue 2

Received Date: July 23, 2018

Published Date: July 26, 2018

\section{Abstract}

This paper tries to help physicians involved in neuromodulation techniques in achieving better results. Patient selections, trial period as well as the neuromodulation technique selection itself are discussed. Initial stimulation parameters for spinal cord stimulation, motor cortex stimulation and peripheral nerve field stimulation are suggested in the text. Neuromodulation techniques must be used properly since its misusage may lead them to failure and to become less accepted into the medical community.

Keywords: Neuromodulation; Peripheral Nerve Field Stimulation; Motor Cortex Stimulation; Spinal Cord Stimulation; Neuropathic Pain

Abbreviations: NM: Neuro Modulation; SCS: Spinal Cord Stimulation; DBS: Deep Brain Stimulation; MCS: Motor Cortex Stimulation; PFS: Peripheral Nerve Stimulation; PNFS: Peripheral Nerve Field Stimulation.

\section{Introduction}

The International Neuromodulation Society defined neuromodulation (NM) as the therapeutic alteration of activity in the central, peripheral or autonomic nervous systems, electrically or pharmacologically, by means of implanted devices. Thus, there must be some device implanted like stimulators or intrathecal pumps [1,2]. Low frequency electrical stimulation has an excitatory effect and it is used in spinal cord stimulation (SCS), periaqueductal gray matter activation by deep brain stimulation (DBS) and during motor cortex stimulation (MCS), peripheral nerve stimulation (PFS) or peripheral nerve field stimulation (PNFS). On the other hand, high frequency stimulation is used to achieve neuronal inhibition in NM techniques such as thalamic and hypothalamic stimulation through DBS [1,3-5]. This manuscript is focused on electrical NM instead of pharmacological one. Although NM is applied to treat several diseases like movement disorders and epilepsy, among others, pain treatment is one of the most common usages. Different types of NM may be chosen such as MCS, PNS, PNFS, DBS, but the most indicated one is SCS. This paper tries to help physicians involved in these techniques in achieving better results.

\section{Patient Selection}

Patient selection is one of the most important issues in order to achieve better outcomes with these techniques. Each case must be evaluated individually since only a minority of the patients who suffers from pain is actually candidates for NM treatments.

The main indication for NM in pain treatment is refractory neuropathic pain, except for PNFS, in which nociceptive pain may be also treated. Nociceptive pain is a physiological response to a stimulus, which warns the person of imminent or actual tissue damage, whereas 


\section{Anaesthesia \& Critical Care Medicine Journal}

neuropathic pain results from direct damage to the nervous system. This structural damage at any point of the sensory pathways (not only in the peripheral, but also in the central nervous system) produces deafferentation pain. Neuropathic pain may also be functional and it happens when a nociceptive pain became chronic and adds clinical features of neuropathic pain through central sensitization. The latter type of pain has a combination of negative (partial or complete loss of sensation: hypo/analgesia, hypo/anesthesia) and positive (dysaethesia, allodynia, hyperalgsia, hyperpathia, paraesthesia and the pain itself) sensory symptoms. The pain itself may be paroxysmal, continuous or both and it can be shooting, lancinating, or burning. Some patients describe their pain as aching and dull [1,5-9]. It is essential to accomplish an accurate diagnosis and to understand pain's pathophysiology and its etiology in order to select the candidates for NM properly. Furthermore, the pain must be refractory to neuropathic pain pharmacological treatment (anticonvulsants and antidepressant), as well as physical therapy. It is optimal to have an institutional protocol of chronic and neuropathic pain treatment in order to establish which drugs would be used as first, second and third tier and which patients would be declared refractory.

\section{Neuromodulation Technique Selection}

Each NM technique has its own indications; however, it must be borne in mind that we need viable neural tissue to stimulate (excitatory or inhibitory) and thus, we should implant the electrode proximally to the lesion in the sensory pathway. Peripheral nerve injury may be treated with PNS, PNFS or SCS; the latter is the best one when multiple nerves are involved. When pain is originated in a nerve root injury, the pain physician must determine if this lesion is proximal or distal to the dorsal root ganglion. If the lesion is proximal to this neural structure, it should be treated like a spinal cord injury because there will be degeneration of the nerve fibers proximal to their cell bodies. It is essential to remember that these fibers will form the dorsal columns within the spinal cord. If the lesion is distal to the ganglion, neuroma formation is common and central sensory fibers in the dorsal columns are intact. Thus, SCS may be utilized. Spinal cord injury may be complete or not and it can be evaluated clinically (complete absence of sensory function below the lesion) and with magnetic resonance images, but it may be necessary to undergo somatosensory evoked potentials with central conduction time test to evaluate if the sensory pathway in the dorsal columns is enough functional to allow using SCS. When the etiology of pain is a partial spinal cord injury (or a partial nerve root lesion proximal to the dorsal root ganglion; see above) and the dorsal columns have enough functional value, SCS may be used for pain treatment. However, in cases with complete spinal cord damage (or a complete nerve root injury located proximal to the dorsal root ganglion), SCS should not be tried and DBS or MCS may be utilized. When certain type of NM fails to achieve adequate pain relief, a more proximal NM technique may be tried, such as DBS or, more common nowadays, MCS. Furthermore, a hybrid construct may be implanted like an SCS electrode plus an MCS electrode, both of them connected to the same implantable pulse generator (IPG) $[1,7,10-12]$.

Despite the absence of good evidence about its better outcomes, MCS has displaced DBS in pain treatment. Thus, DBS is rarely used for this indication nowadays. MCS has certain indications, such as: central post stroke pain, neuropathic trigeminal pain, brachial plexus avulsion (principally for continuous pain since paroxysmal pain is managed better with Dorsal Root Entry Zone lesioning) and infrasegmental pain in spinal cord injury. Segmental pain in spinal cord injury is preferentially treated with Dorsal Root Entry Zone lesioning than MCS [11,13-19].

\section{Trial}

Commonly, a trial period in which the electrodes are connected to disposable extensor leads is performed in different NM techniques (SCS, MCS, PNS, PNFS and DBS). Other NM techniques like vagal nerve stimulation do not include it. An external pulse generator is used to stimulate the electrodes through the disposable extensor lead. This kind of generators allows the patients and their families to turn it on or off as well as controlling the stimulation amplitude. It must be borne in mind that some NM modalities, such as classical SCS, thalamic DBS, PNFS and PNS, produces paresthesia in the stimulated dermatomas, whereas other types of NM like MCS and burst or high frequency SCS do not elicit paresthesia. An ambulatory trial is the gold standard and although it can last from three days to three weeks, it is not commonly recommended to be longer than a week because of the infection risk. The positive response to the trial is generally established as a pain relief of at least 50\% according to the visual analog scale (VAS) as well as patient satisfaction $[1,5,14,15,20]$.

The trail period is not only a tool of the pain physician to improve patient selection, but also an element to increase the cost-effectiveness of the techniques, since the IPG is the most expensive component of the NM systems and it will only be implanted in those patients who present a positive trial. In those patients who present a 


\section{Anaesthesia \& Critical Care Medicine Journal}

negative trial (nonresponders), permanent implantation of the IPG is contraindicated and the electrode should be removed [1]. Regarding to the initial stimulation parameters, in SCS we usually apply: frequency $=60 \mathrm{~Hz}$, pulse width $=110-170 \mu$ seg and the intensity needed to produces paresthesia in the pain area, but without eliciting unpleasant disesthesia. We use bipolar stimulation and sometimes complex contact configurations are needed to achieve paresthesia coverage as well as pain relief. During the initial stimulation session in MCS, we use also bipolar stimulation between two contacts with the following parameters: frequency $=30-60 \mathrm{~Hz}$, pulse width $=200-350$ $\mu$ seg. We start with an intensity of $1 \mathrm{~mA}$ and then, if we need it, it is increased until reach $70-80 \%$ of the motor threshold. In PNFS, the initial stimulation parameters are more heterogeneous since very different electrode configurations with variably distances between them can be implanted; however, we commonly start an excitatory stimulation with a frequency of $30 \mathrm{~Hz}$ and a pulse width of approximately $200 \mu$ seg. The pain physician who performs NM devices implantation must be involved in the programming sessions in order to reach a better knowledge of the clinical cases, the NM devices and the techniques itself.

\section{Conclusion}

NM techniques are essential in refractory neuropathic pain management nowadays. However, these techniques must be used properly since its misusage may lead them to failure and to become less accepted into the medical community.

\section{References}

1. Yampolsky C, Hem S, Bendersky D (2012) Dorsal column stimulator applications. Surg Neurol Int 3(4): 275-289.

2. Bendersky D, Yampolsky C (2014) Is Spinal Cord Stimulation Safe? A Review of Its Complications. World Neurosurg 82(6): 1359-1368.

3. Hatzis A, Stranjalis G, Megapanos C, Sdrolias PG, Panourias IG, et al. (2007) The current range of neuromodulatory devices and related technologies. Acta Neurochir Suppl 97(1): 21-29.

4. Velasco F, Velasco AL, Núñez JM, Castro G, CarrilloRuiz JD, et al. (2010) Neuromodulation: History, principles and current trends. In: Cukiert A, editor. Neuromodulation. Sao Paulo: Alaude Editorial 22-36.
5. Cairnsak, Porter McRoberts W, Deer T (2011) Peripheral Nerve Stimulation for the Treatment of Truncal Pain. Prog Neurol Surg 24: 58-69.

6. Woolf C, Mannion R (1999) Neuropathic pain: aetiology, symptoms, mechanisms, and management. Lancet 353(9168): 1959-1964.

7. Gonzalez Machado J (2002) Dolor neuropático, Taxonomia. In: Villoria C, (Eds.), Dolor Neuropático. Reunión de Expertos. Salamaca: Fundacion Grunenthal pp: 17-26.

8. Martínez-Salio A, Gámez De La Cámara A, Ribera Canudas M, Montero Homs J, BlancoTarrío E, et al. (2009) Diagnosis and treatment of the neuropathic pain. Med Clin (Barc) 133(16): 629-636.

9. Sandkühler J (2009) Models and Mechanisms of Hyperalgesia and Allodynia. Physiol Rev 89(2): 707 758.

10. Sindou M, Mertens P, Bendavid U, Garcia-Larrea L, Mauguiere F (2003) Predictive value of somatosensory evoked potentials for long-lasting pain relief after spinal cord stimulation: practical use for patient selection. Neurosurgery 52(6): 1374-1383.

11. Nguyen J-P, Nizard J, Keravel Y, Lefaucheur J-P (2011) Invasive brain stimulation for the treatment of neuropathic pain. Nat Rev Neurol 7(12): 699-709.

12. Atkinson L, Sundaraj S, Brooker C, O'Callaghan J, Teddy P, et al. (2011) Recommendations for patient selection in spinal cord stimulation. J Clin Neurosci 18(10): 1295-1302.

13. Sindou M (2009) Neurosurgery for Neuropathic Pain. In: Sindou M, editor. Practical Handbook of Neurosurgery. Germany: Springer-Verlag / Wienp 3: 295-316.

14. Machado A, Mogilner A, Rezai A (2009) Motor Cortex Stimulation for Persistent Non-Cancer Pain. In: Lozano A, [Eds.], Textbook of stereotactic and functional neurosurgery. Berlin: Springer-Verlag pp: 2239-2250.

15. Hamani C, Fontaine D, Lozano A (2009) DBS for Persistent Non-Cancer Pain. In: Lozano A, (Eds.), Textbook of stereotactic and functional neurosurgery. Berlin: Springer-Verlag pp: 2227-2238. 


\section{Anaesthesia \& Critical Care Medicine Journal}

16. Klit H, Finnerup N, Jensen T (2009) Central poststroke pain: clinical characteristics, pathophysiology, and management. Lancet Neurol 8(9): 857-868.

17. Pagano RL, Fonoff ET, Dale CS, Ballester G, Teixeira MJ, et al. (2012) Motor cortex stimulation inhibits thalamic sensory neurons and enhances activity of PAG neurons: possible pathways for antinociception. Pain 153(12): 2359-2369.

18. Henderson J, Lad S (2006) Motor cortex stimulation and neuropathic facial pain. Neurosurg Focus 21(6): 6.
19. Zaghi S, Heine N, Fregni F (2009) Brain stimulation for the treatment of pain: A review of costs, clinical effects, and mechanisms of treatment for three different central neuromodulatory approaches. J Pain Manag 2(3): 339-352.

20. Al-Kaisy A, Van Buyten JP, Smet I, Palmisani S, Pang D, et al. (2014) Sustained Effectiveness of $10 \mathrm{kHz}$ HighFrequency Spinal Cord Stimulation for Patients with Chronic, Low Back Pain: 24-Month Results of a Prospective Multicenter Study. Pain Medicine 15(3): 347-354.

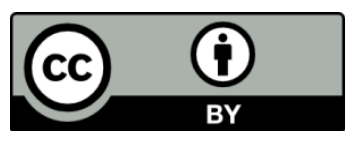

